\title{
E-cigarettes: A new hazard for children and adolescents
}

\author{
Sarah A. Richmond $\mathrm{PhD}^{1}$, Ian Pike $\mathrm{PhD}^{2,3}$, Jonathon L. Maguire $\mathrm{MD}^{4,5}$, Alison Macpherson $\mathrm{PhD}^{1}$ \\ ${ }^{1}$ School of Kinesiology \& Health Science, York University, Toronto, Ontario; ${ }^{2}$ Department of Pediatrics, University of \\ British Columbia, Vancouver, British Columbia; ${ }^{3}$ British Columbia Injury Research and Prevention Unit, Child and \\ Family Research Institute at BC Children's Hospital, Vancouver, British Columbia; ${ }^{4} \mathrm{Li} \mathrm{Ka}$ Shing Knowledge Institute, \\ St. Michael's Hospital, Toronto, Ontario; ${ }^{5}$ Department of Pediatrics, University of Toronto, Toronto, Ontario
}

Correspondence: Sarah A. Richmond, School of Kinesiology \& Health Science, 339 Bethune College, York University, 4700 Keele St., Toronto, Ontario M3J 1P3. Telephone 416-736-2100 x 77211, e-mail sarah.a.richmond@gmail.com

\section{Abstract}

Objectives: Electronic cigarettes and fluid (e-cigarettes, e-fluid) are hazardous materials that when inhaled or ingested may pose significant health risks to children and adolescents. The objective of this work was to explore the spectrum of injury related to e-cigarette exposure among Canadian children and adolescents.

Methods: A one-time survey was sent to all paediatricians in Canada. Information was collected on children and adolescents who presented with e-cigarette exposure (inhalation and ingestion cases) in the previous 12 months. Questions included the number of injuries and symptoms, in addition to age, sex, treatment setting, intentional e-cigarette use and how the products were accessed.

Results: A total of 520 surveys were completed and returned, identifying 220 cases. Symptoms related to inhalation were present in 135 cases (43 unintentional, 92 intentional) and in 85 ingestion cases ( 35 unintentional, 50 intentional). For inhalatiobcases, most were male, aged 15 to 19 years, who sought treatment for nausea/vomiting, cough, throat irritation or acute nicotine toxicity in an outpatient clinic/office. Most inhalation cases reported e-cigarette use 2 to 3 days/week, and e-cigarettes purchases from a mall kiosk/store. For ingestion cases, most were male, aged 1 to 4 years presenting to an emergency department with nausea/vomiting, cough or respiratory irritation. Younger cases accessed e-fluid at home, older cases purchased in a mall kiosk/store. E-fluid flavours reported consumed were fruit, candy, and tobacco.

Conclusions: E-cigarettes, recently introduced into the North American market are hazardous to children and adolescents. Further investigation into the risks that e-cigarettes pose and ways to reduce exposure is needed to minimize injury.

Keywords: Electronicnicotine delivery systems; Wounds and injuries; Child; Adolescent.

Children and adolescents represent a vulnerable population in that even small exposures to hazards, particularly ingested hazards, can cause injury or death. Injury prevention efforts in the 1960 's and 1970's made significant improvement to morbidity and mortality from unintentional poisoning by implementing product safety standards including recommendations about safe storage, packaging, handling and disposal of prescription and over the counter medication, and household cleaning products (e.g., antifreeze) (1). Despite these successes, unintentional poisoning is one of the only injury types in North
America that has demonstrated annual increases in hospitalizations and deaths (2-4). Children under the age of 6 years are most frequently exposed to potential toxins, representing 52\% of all exposures $(2,4)$. This may be due to the ever increasing availability of potentially toxic substances in the market that are available and accessible to children.

Electronic cigarettes (e-cigarettes) are a type of electronic nicotine delivery system that when activated, vaporizes and delivers inhalable liquid (e-fluid). The e-fluid contains glycerin, propylene glycol, flavourings and other substances (5) and may or may 
not contain nicotine in varying concentrations. These materials, when inhaled and/or ingested, pose significant health risks to children and adolescents, including nicotine, a major ingredient found in many e-fluid solutions. Of particular importance with the nicotine found in e-fluids are variations in concentration per refill solution and the significant discrepancy between actual and labelled concentrations (6,7). In Canada and the USA, it is not required by law to meet standards to label the amount of nicotine contained in the e-fluid mixtures $(8,9)$. In addition to nicotine, other ingredients found in e-cigarette devices are harmful to child health and include glycerin, propylene glycol, aldehydes, metals, tobacco-specific alkaloids and polycyclic aromatic hydrocarbons (10). The most popular solvents found in e-fluid are glycerin and propylene glycol (11). When the user initiates inhalation of the e-cigarette device, the heating element raises the temperature of the e-fluid, which in turn, activates changes to the composition of the liquids, forming potentially toxic compounds including formaldehyde and acetaldehyde (11). Formaldehyde and acetaldehyde are widely published as Group 2A and 2B carcinogens, respectively (12). A recent study measured the carbonyl compounds from commercially available e-fluids and found formaldehyde and acetaldehyde in 8 of the 13 samples, with levels of formaldehyde, acetaldehyde and acetone comparable to tobacco in high-voltage devices (11). Nicotine concentrations of $10 \mathrm{mg}$ is considered a lethal dose in child and adolescent exposures (a dose of 6.5 to $13 \mathrm{mg} / \mathrm{kg}$ is fatal in adults) $(6,13,14)$; considering the average e-fluid refil container of $10 \mathrm{~mL}$ with a reported $20 \mathrm{mg} / \mathrm{mL}$ level of nicotine, ingestion of this fluid by a child would exceed lethal concentrations $(6,13)$. In a recent study by Davis et al., nicotine concentrations assessed in over 70 e-cigarette refill flyids ranged from 0 to $134 \mathrm{mg} / \mathrm{mL}$ (6). The rise in popularity of these devices has raised many unanswered questions about their impact on children and adolescents. Data from the 2013 Canadian Tobacco, Alcohol and Drugs Survey (CTADS) indicated that approximately 2.5 million Canadians ages 15 years and older reported having experimented with an e-cigarette device (15). One in 5 youth ages 15 to 19 years as well as young adults ages 20 to 24 years reported at least one use of an e-cigarette; the highest reported use across all age groups (15). Data from middle school students who completed the 2013 National Youth Tobacco Survey in the USA, demonstrated increased use from $1.4 \%$ in 2011 to $3 \%$ in 2013 among those who reported having ever used an e-cigarette (16). In Canada, $14.6 \%$ of secondary students that responded to the Ontario Student Drug Use and Health Survey (OSDUHS) in 2013 reported lifetime e-cigarette use; in 2015 , the proportion increased to $28.2 \%$ (17). In addition, despite legislation in Canada that restricts e-cigarette products from containing nicotine (18), 18.8\% of OSDUHS regular use respondents reported use of a nicotine-containing e-cigarette $(17,19)$. In addition to the known adverse health effects of nicotine and other toxic exposures found in e-fluid for youth, the potential risk of serious injury or death among children from poisoning from contact with the e-fluid is high (20). Canadian and US studies have reported an increase in calls to poison centres due to exposure to e-cigarette products, particularly ingestion of the e-fluid in younger children (21-25).

More information on the frequency of child and adolescent exposure to these devices and their content is needed in order to understand the underlying risk. There is minimal data 1 in Canada and the USA on the adverse affects of e-cigarette exposure among youth. In particular, there is minimal information on the unintentional ingestion of e-fluid (often flavoured with candy, chocolate, alcohol and fruit, for example) used for refilling the device. In addition, muck of the information on potentially adverse exposures to e-cigarettes among children and adolescents comes from poison centres, and much of this information focuses on young children. Descriptions of potentially adverse exposures among older children, particularly adolescents, are lacking. Moreover, much of the literature on e-cigarettes is from the United States and Europe. The aim of this study was to describe the spectrum of injuries observed by Canadian paediatricians related to inhalation of e-cigarettes or ingestion of e-cigarette fluid among children and youth.

\section{METHODS}

A cross-sectional study was conducted by administering a one-time survey (https://www.cpsp.cps.ca/uploads/surveys/ e-cigarettes-e-liquid-survey-questions.pdf) to all practicing paediatricians participating in the Canadian Paediatric Surveillance Program (CPSP) $(n=2533)$. The CPSP conducts national surveillance on paediatric disorders or conditions that are high in disability, morbidity, mortality and economic cost to society, despite low frequency. The CPSP gathers information through multi-year studies and one-time surveys. Participants were asked about the number and injuries/symptoms of children and adolescents who had presented with e-cigarette exposure (inhalation and ingestion cases) in the previous 12 months. In addition, information was collected on patients' age, sex, treatment setting sought, e-cigarette use and access.

Exposure was categorized into either ingestion or inhalation cases. Questions for cases included patient age group ( $<1$ year, 1 to 4 years, 5 to 9 years, 10 to 14 years, 15 to $<19$ years) and sex (male, female). Health care setting was categorized as office or outpatient clinic, hospital inpatient ward, hospital emergency department or other (specify). Injury and/or symptom categories included nausea/vomiting, cough, throat irritation, mouth irritation, respiratory irritation, symptoms of acute nicotine toxicity (other than nausea/vomiting), burn (skin, mouth/ esophagus), decrease in pulmonary function (e.g., increased dynamic airway resistance), central nervous system depression, 
other (specify). Access to the e-fluid was categorized by purchases from the Internet, from a mall kiosk/convenience store, in the home, from parents/caregivers, friends, unknown or other (specify). Finally, respondents were asked to indicate the types of flavours most commonly reported ingested from the following: tobacco, fruit, coffee, menthol, candy, alcohol, unknown and other (specify). Respondents were also asked to provide the frequency of reported use of e-cigarettes in inhalation cases that included: once, once per week, two to three times per week, four to seven times per week and more than seven times per week.

All data were analyzed using STATA 12.0 (StataCorp, Texas) software and aggregate descriptive data are reported. This study did not require research ethics approval due to the aggregate and anonymous collection of data for surveillance purposes.

\section{RESULTS}

Of the 2533 paediatricians and paediatric subspecialists in Canada, 520 surveys were received, representing a 20.5\% response frequency. Twenty per cent of respondents indicated that the topic presented in the survey was not relevant to their area of practice. Over the previous year period, there were 220 children and adolescents under the age of 19 years, seen by paediatricians for injuries/symptoms from exposure to e-cigarettes. Fifty per cent were seen by a general paediatrician and $50 \%$ by a paediatric subspecialist. Of the cases seen by subspecialists, most were emergency medicine, adolescent medicine, gastroenterology or infectious disease experts. Symptoms related to inhalation were present in 135 cases (43 unintentional, 92 intentional) and in 85 cases related to ingestion (35 unintentional, 50 intentional) (see Table 1 for characteristics by ingestion and inhalation case type).

For inhalation cases, the majority of cases were reported to be male, ages 15 to 19 years. Cases most often sought treatment for cough, nausea/vomiting, throat irritation or acute nicotine toxicity in an outpatient clinic or office. Most inhalation cases reported e-cigarette use 2 to 3 days per week and e-cigarettes purchases from a mall kiosk/store. For ingestion cases, most were male, ages 1 to 4 years presenting to an emergency department with nausea/vomiting, cough or respiratory irritation. Younger ingestion cases accessed e-fluid at home older cases purchased it in a mall kiosk/store. E-fluid flavours reported consumed were fruit, candy and tobacco.

\section{Discussion}

The results of this survey suggest that e-cigarette products cause injury to a number of Canadian children and youth. E-cigarettes have increased in popularity and accessibility in Canada in recent years (8). What remains unknown is the adverse affects of e-cigarettes among children and youth, including exposure from inhalation and ingestion of the associated products (i.e., vapou and e-fluid). In 2009, Health Canada provided guidelines for the importation and sale of e-cigarettes and related products that contained nicotine. What remained; however, was the ability for consumers of all ages to purchase e-cigarettes and related products, particularly over the internet, and these products have been cited to have significant variability in the actual nicotine content in the e-fluid $(6,7)$. Legislation to control the sale and use of e-cigarettes at the federal level has not been passed; however, there is some regulationat the provincial and territorial level in Canada. Provinces in Canada that have passed legislation to include e-cigarettes in the Tobacco Sale and Access Act that include restrictions on the sale or purchase of e-cigarette and e-cigarette products include Ontario (January, 2016 - passed), British Columbia (March, 2015 - introduced), Manitoba (November 2015 - passed), Nova Scotia (May, 2015 - passed), New Brunswick (July, 2015 - passed), Quebec (November, 2015 - passed) and Prince Edward Island (June, 2015 - introduced). Provinces and territories yet to introduce provincial legislation include Saskatchewan, North west Territories, Yukon, Nunavut, Newfoundland \& Labrador, and Alberta. Of the provinces that have enacted legislation, all provinces except Ontario have also included legislation to include e-cigarettes in the Smoke-Free Places Act that restricts use of e-cigarettes in specified locations (e.g., bars, restaurants, hotels, etc.).

Regulation of e-cigarettes and e-fluid contents and packaging are also issues of concern in Canada. The liquid used in e-cigarette devices is often flavoured with candy and fruit; flavouring that may be appealing to the first-time, experimental user. In addition, the labelling for e-fluids is often colourful, which may draw the attention of younger children who are unaware of the contents of the product, placing them at risk of unintentional ingestion. From this survey, 34 cases presented to a health care setting as a result of unintentional ingestion of e-fluids. Unintentional ingestion of e-fluid is of serious concern as labelling is not required to meet

Table 1. Aggregate* patient characteristics

\begin{tabular}{lll}
\hline Age & Inhalation cases $(\mathrm{n}=135)$ & Ingestion cases $(\mathrm{n}=85)$ \\
Most reported injuries/symptoms & $15-19$ years & $1-4$ years \\
Setting patient treated & Cough, nausea/vomiting & Nausea/vomiting, cough \\
E-cigarette use & Outpatient clinic/office & Emergency department \\
E-cigarette access & $2-3$ times/week & N/A \\
\hline
\end{tabular}

${ }^{*}$ Data shown represents characteristics for the majority of cases presented in each category (inhalation vs. ingestion). N/A Not applicable. 
any restrictive or legal standards in Canada (8). In addition, there is no legislation to label the actual nicotine content in e-fluids, and it has been reported that these concentrations vary widely $(6,7)$. Finally, there have been several reports in mainstream media of e-cigarette devices causing serious injury by exploding. In the October 2014 report from the Federal Emergency Management Agency (FEMA) in the USA, there were 25 separate incidents of explosion and fire involving an e-cigarette device reported in the media (26). In a recent review of all media sources found on local and national sites on the Internet, there have been a total of 183 reports of e-cigarette explosions (27).

The results of this survey are consistent with the existing literature. For both inhalation and ingestion, the majority of cases were reported to be male. This is consistent with self-reported data from the Ontario Student Drug Use and Health Survey that reported males (14.5\%) were significantly more likely to use e-cigarettes, compared to females (8.7\%) (17). In addition, a recent study by Reid et al. found Canadian male teens to have slightly higher self-reported use of e-cigarettes over females (28). Data from the USA also supports higher use among males; $15 \%$ of males compared to $11.9 \%$ of females reported use of an e-cigarette in the previous 30 days $(16,29)$ and Forrester et al. reported $51 \%$ of cases reported to Texas poison centres were male (24). This is the first study in Canada to report the adverse health effects of exposure to e-cigarettes and e-fluid in children and adolescents. Studies from the US cite similar reported injuries including cough, mouth and throat irritation (30-33); however, these studies were reported adult e-cigarette users. One US study in children under the age of 5 , reported the most commonlyreported clinical effects of cases included vomiting, drowsiness and cough (24). US poison control centres have reported a 28 fold increase, ( 84 cases in 2011 to 2360 cases in 2014) in the number of calls about children ages 5 and under who had been exposed to e-fluid containing nicotine (34), and an increase in the number of cases of acute nicotine toxicity from unintentional exposures to e-fluid, including one reported child death (20).

\section{Conclusions}

E-cigarettes, recently introduced into the Canadian market are causing harm to children and adolescents. Results of this study highlight the spectrum of injuries resulting from exposure to e-cigarettes and e-fluid. Children and youth reported in this survey pressented to paediatricians with injuries including cough, hausea/vomiting, throat and respiratory irritation or acute nicotine toxicity. Improving education for parents and caregivers about the risk of injury that can result from exposure to e-cigarettes and e-fluid may reduce the risk. However, more stringent legislation around the use, access, and control of sale and purchase of e-cigarettes in Canada and the USA is needed. Policy recommendations from the Canadian Paediatric Society related to the federal Tobacco Control Act include: regulation of the marketing, packaging (including child resistant packaging on all e-fluid containers) and labelling of devices (i.e., enforcement of a maximum dose of nicotine in e-fluids together with accurate labelling; all packaging to include information of the potential and known harmful effects of use; banning advertising, sponsorship and marketing at events and activities where children and youth are involved); restriction of e-cigarette manufacturers or sellers from making positive health claims related to the use $\mathrm{O}$ the products; restrictions on the sale of e-cigarettes and products over the internet to identified adults with direct mailing options; and enactment of legislation, across all provinces and territories, making it illegal for children and youth (by provincial legal age determinants) to use, purchase or have any form of e-cigarette device (8). The AmericanAssociation for Cancer Research, the American Society of Clinical Oncology and the American Academy of Pediatries have called for regulation in the USA, recommending that all levels of government regulate the sale, labelling, advert sing and packaging of e-cigarette and related products $(20,35)$. Further investigation into the health impacts, patterns of use, access and mechanisms to reduce the risks that e-cigarettes pose to children and adolescents is needed in order to minimize injury and to provide the evidence to support provincial and federal advocacy for policy change. Finally, Canadians should follow the progress being made by the recent introduction of Bill S-7, first read into parliament on November 22, 2016 (36). This Bill proposes to amend the existing Tobacco Act and the Non-smokers Health Act to regulate vaping and vaping products under the newly renamed Act, the Tobacco and Vaping Products Act (36). This Bill does not include all of the recommendations provided in this paper; however, it is a step in the right direction to regulate these hazardous products with respect to selling, packaging, labelling and use.

\section{Acknowledgments}

We would like to acknowledge all of the participating paediatricians in Canada for taking part on this survey. In addition, we would like to thank the Canadian Paediatric Surveillance Program, a partnership between the Canadian Paediatric Society and the Public Health Agency of Canada.

\section{Conflict of Interest}

AM reports grants from Canadian Institutes of Health Research during the conduct of the study.

\section{References}

1. Warner M, Chen LH, Makuc DM, Anderson RN, Miniño AM. Drug poisoning deaths in the United States, 1980-2008. NCHS Data Brief. 2011;81:1-8.

2. Chen $Y$, Mo F, Yi QL, Jiang Y, Mao Y. Unintentional injury mortality and external causes in Canada from 2001 to 2007. Chronic Dis Inj Can 2013;33(2):95-102. 
3. Bond GR, Woodward RW, Ho M. The growing impact of pediatric pharmaceutical poisoning. j Pediatr 2012;160(2):265-270.e1.

4. British Columbia Injury Research and Prevention Unit (BCIRPU) [Internet]. British Columbia: Poison quick facts. Cited April 30, 2016. Available from: http://www.injuryresearch.bc.ca/ quick-facts/poisoning/.

5. Etter JF, Zäther E, Svensson S. Analysis of refill liquids for electronic cigarettes. Addiction 2013;108(9):1671-9.

6. Davis B, Dang M, Kim J, Talbot P. Nicotine concentrations in electronic cigarette refill and do-it-yourself fluids. Nicotine Tob Res 2015;17(2):134-41.

7. Goniewicz ML, Kuma T, Gawron M, Knysak J, Kosmider L. Nicotine levels in electronic cigarettes. Nicotine Tob Res 2013;15(1):158-66.

8. Stanwick R. E-cigarettes: Are we renormalizing public smoking? Reversing five decades of tobacco control and revitalizing nicotine dependency in children and youth in Canada. Paediatr. Child Heal. 2015;20(2):101-5.

9. US Department of Health and Human Services. US Drug and Food Administration [Internet]. Silver Springs: Electronic cigarettes (e-cigarettes). Updated May 9, 2016; cited Jun 2, 2016. Available from: http://www. fda.gov/NewsEvents/PublicHealthFocus/ucm172906.htm.

10. Cheng T. Chemical evaluation of electronic cigarettes. Tob Control. 2014;23(Suppl 2):ii11-iil7.

11. Kosmider L, Sobczak A, Fik M, et al. Carbonyl compounds in electronic cigarette vapors: Effects of nicotine solvent and battery output voltage. Nicotine Tob Res 2014;16(10):1319-26.

12. World Health Organization, International Agency for Research on Cancer. IARC Monographs on the Evaluation of Carcinogenic Risks To Humans: Volume 83, Tobacco Smoke and Involuntaly Smoking. Geneva, 2004. Available from: http://monographs.larc. fr/ENG/Monographs/vol83/mono83-1.pdf.

13. Mayer B. How much nicotine kills a human? Trácing back the generally accepted lethal dose to dubious self-experiments in the nineteenth century. Arch Toxicol 2014;88(1):5-7.

14. Etter JF, Bullen C, Flouris AD, Laugesen M, Eissenberg $T$. Electronic nicotine delivery systems: A research agenda. Tob Control 2011;20(3):243-8.

15. Statistics Canada. [Internet] Ottawa: Canadian Tobacco, Alcohol, and Drugs Survey (CTADS). Updated Feb 3, 2015; cited Jun 2, 2016. Available from: http://healthycanadians.gc.ca/science-research-sciences-recherches/data-donnees/ctads-ectad/summary-sommaire-2013-eng.php.

16. Arrazola RA, Singh T, Corey CG, et al. Tobacco use among middle and high school students - United States, 2011-2014. mmwr Morb Mortal Wkly Rep 2015;64(14):381-5.

17 Boak A, Hamilton H, Adlaf E, Mann R. Drug Use Among Ontario Students, 1977-2015: OSDUHS Highlights. Toronto,

ON: Centre for Addiction and Mental Health; 2015.

18. Czoli C, Reid J, Rynard VL, Hammond D. E-Cigarettes in Canada - Tobacco Use in Canada: Patterns and Trends, 2015 Edition, Special Supplement; 2015. Available at: http://www.tobaccoreport. ca/2013/TobaccoUseinCanada_2013.pdf.

19. Hamilton HA, Ferrence R, Boak A, et al. Ever use of nicotine and nonnicotine electronic cigarettes among high school students in Ontario, Canada. Nicotine Tob Res 2015;17(10):1212-8.
20. Walley S, Jenssen B. Electronic nicotine delivery systems. Pediatrics. 2015;136(5):1018-26.

21. Kim JW, Baum CR. Liquid nicotine toxicity. Pediatr. Emerg. Care. 2015;31(7):517-21.

22. Gill N, Sangha G, Poonai N, Lim R. E-cigarette liquid nicotine ingestion in a child: Case report and discussion. CJEM. 2015:1-5.

23. Vakkalanka JP, Hardison LS Jr, Holstege CP. Epidemiological trends in electronic cigarette exposures reported to u.s. Poison centers. Clin Toxicol (Phila) 2014;52(5):542-8.

24. Forrester MB. Pediatric exposures to electronic cigarettes reported to Texas poison centers. j Emerg Med 2015;49(2)

25. Gupta S, Gandhi A, Manikonda R. Accidenta/nicotine liquid ingestion: Emerging paediatric problem. Arch Dis Child 2014;99(12):1149.

26. Federal Emergency Management Agency. US Department of Homeland Security. [Internet]. New York: Electronic Cigarette Fires and Explosions. Cited October 4, 2016. Available at: https://www.usfa.fema.goy/downloads/pdf/publications/electronic_cigarettes.pdf.

27. Ecigone.com [Internet]. E-Cigarette Explosions: Comprehensive List (updated July 13, 2016; cited October 4, 2016. Available at: http:// ecigone.com/featured/e-cigarette-explosions-comprehensive-list/.

28. Reid JL, Rynard VL, Czoli CD, Hammond D. Who is using e-cigarettes in Canada? Nationally representative data on the prevalence of e-cigarette use among Canadians. Prev. Med. (Baltim). 2015;81:180-3.

29. Lippert AM. Do adolescent smokers use e-cigarettes to help them quit? The sociodemographic correlates and cessation motivations of u.s. adolescent e-cigarette use. Am J Health Promot 2015;29(6):374-9.

30. Bhatnagar A, Whitsel LP, Ribisl KM, et al. Electronic cigarettes: A policy statement from the American Heart Association. Circulation. 2014;130(16):1418-36.

31. Bullen C, McRobbie H, Thornley S, Glover M, Lin R, Laugesen M. Effect of an electronic nicotine delivery device (e cigarette) on desire to smoke and withdrawal, user preferences and nicotine delivery: Randomised cross-over trial. Tob Control 2010;19(2):98-103.

32. Flouris $\mathrm{AD}$, Chorti MS, Poulianiti KP, et al. Acute impact of active and passive electronic cigarette smoking on serum cotinine and lung function. Inhal Toxicol 2013;25(2):91-101.

33. Goniewicz ML, Lingas EO, Hajek P. Patterns of electronic cigarette use and user beliefs about their safety and benefits: An internet survey. Drug Alcohol Rev 2013;32(2):133-40.

34. Mowry JB, Spyker DA, Cantilena LR Jr, Bailey JE, Ford M. 2012 Annual Report of the American Association of Poison Control Centers' National Poison Data System (NPDS): 30th Annual Report. Clin Toxicol. 2013;51(10):949-1229.

35. Brandon TH, Goniewicz ML, Hanna NH, et al. Electronic nicotine delivery systems: A policy statement from the American association for cancer research and the American society of clinical oncology. j Clin Oncol 2015;33(8):952-63.

36. Senate of Canada. [Internet]Bill S-5: An Act to Amend the Tobacco Act and the Non-smokers' Health Act and to Make Consequential Amendments to Other Acts. Updated April 4, 2017; cited May 23, 2017. Available at: http://www.parl.ca/DocumentViewer/ en/42-1/bill/S-5/first-reading. 